\title{
Simulation and Analysis of Microtremor H/V Spectrum Based on the Surface Wave Propagation Theory in Layered Half-Space
}

\author{
Xueliang Chen ${ }^{1, *}$ Zhen Zhang ${ }^{2,1}$ Mengtan Gao ${ }^{1}$ \\ ${ }^{1}$ Institute of Geophysics, China Earthquake Administration, Beijing, China, 100081. \\ ${ }^{2}$ Institute of Mountain Hazards and Environment, Chinese Academy of Sciences, Chengdu, China, 610041. \\ ${ }^{*}$ Corresponding author. Email: xueliang_chen@aliyun.com
}

\begin{abstract}
Subsurface velocity structures can be constructed via an inversion of the horizontal-to-vertical (H/V) spectral ratio of microtremor (MHV) curves, as a result, a method of simulating the MHV curves is key. In this study, we use the H/V spectral ratio of the surface wave (SHV) based on the surface wave propagation theory in layered half-space to simulate the MHV curves at sites A and B of the Yuxi basin. Then, we attempt to analyse the characteristics of the SHV curves. We find the H/V ratio of the microtremor loading source to be independent of the predominant frequency of the SHV curve, but it has some relation to the amplitude of the SHV curve. Moreover, to reduce the error in subsurface velocity structures obtained by the MHV curves, we suggest that the SHV curves at near-predominant frequencies should not be considered in the inversion, because the amplitude deviation is higher at the predominant frequency of the MHV curve.
\end{abstract}

Keywords(Bold): Velocity structure, Microtremor Surface wave H/V spectrum, Microtremor loading source, Simulation and analysis

\section{基于层状半空间面波传播理论之地脉动 $\mathrm{H} / \mathrm{V}$ 谱模拟 分析 \\ 陈学良 ${ }^{1, *}$ 张振 $^{2,1}$ 高孟潭 $^{1}$}

\begin{abstract}
${ }^{1}$ 中国地震局地球物理研究所, 强震动地震学研究室, 北京市海淀区民族大学南路 5 号, 由编：100081
${ }^{2}$ 中国科学院水利部成都山地灾害与环境研究所, 四川省成都市人民南路四段 9 号, 邮编: 610041

*通讯作者. 电子屷箱: xueliang_chen@aliyun.com
\end{abstract}

\begin{abstract}
摘要
地下速度结构可以通过反演地脉动的水平与坚向 (H/V) 傅立叶谱的谱比即（MHV）曲线加以构造，因此，一 种模拟 MHV 曲线的方法是解决该问题的关键。该研究中，我们使用基于表面波在成层半空间中传播理论的表 面波 $\mathrm{H} / \mathrm{V}$ 谱比（SHV 理论方法）来模拟玉溪盆地 A 和 B 站点的地脉动 MHV 曲线。然后, 我们尝试分析 SHV 曲 线的特征。我们发现地脉动加载源的 H/V 傅里叶谱谱比与 SHV 曲线的卓越频率无关, 但与 SHV 曲线的振幅有 一定关系。此外, 为减少由 MHV 曲线获得的地下速度结构中的误差, 我们建议在反演中不考虑近卓越频率 (主频率) 的 SHV 曲线, 因为在 MHV 曲线的卓越频率处振幅偏差较高。
\end{abstract}

关键字: 速度结构, 地脉动面波 $H / V$ 谱, 地脉动加载源, 模拟与分析. 


\section{1. 引言}

大地轻微、连续的振动, 不停地像人的脉搏一 样跳动, 故此得名(袁一凡等, 2012)。地脉动的产生 机理还没有定论。普遍认为地脉动激发源可分为人 为因素和自然因素。在场地特性评价、速度结构探 测以及地震小区划等方面, 地脉动都有大量的应用 (袁一凡等，2012）。

地脉动数据相比于钻孔和地震测线勘探等其他 建模数据, 更加经济和方便(Picozzi et al., 2005)。自 20 世纪 30 年代, 日本相关学者开始对地脉动进行研 究, 关于地脉动的研究已取得了很大的进展。1971 年 Nogoshi 提出利用由单点三分量检波器获取的地 脉动数据计算水平与垂直向地脉动的傅里叶谱 $(\mathrm{H} / \mathrm{V}$ 谱), 单点地脉动 $\mathrm{H} / \mathrm{V}$ 谱也被广泛用于场地效应的评 估(Bonnefoy-Claudet et al. 2006; Carcione et al. 2017)， 特别是, 我国学者在地脉动单点谱比、台阵观测 Rayleigh、Love 面波反演浅层结构、场地类别划分 等方面开展了大量卓有成效的工作（王振东，1986; 杨学林, 吴世明等, 1997; 郭迅, 黄玉龙, 袁一凡, 2000; 陶夏新, 刘曾武, 郭明珠等, 2001; 陶夏新, 师黎静, 董连成, 2002; 郭明珠, 谢礼立, 凌贤长, 2004 ; 师黎静, 陶夏新, 2007; 董连成, 陶夏新, 师黎静等, 2008; 师黎静, 苏茜, 刘宇实等, 2016; Zhang Z, Chen XL, Gao MT, et al, 2018）。

此外, 一些学者尝试通过一维水平地层模型推 导的基阶 Rayleigh 波 $\mathrm{H} / \mathrm{V}$ 谱模拟地脉动 $\mathrm{H} / \mathrm{V}$ 谱(Fäh et al., 2003)。虽然地脉动 $\mathrm{H} / \mathrm{V}$ 谱与基阶 Rayleigh 波 $\mathrm{H} / \mathrm{V}$ 谱的峰频和谷频一致, 但两者的振幅相差较大 (Yamanaka，1994)。也有学者基于扩散场理论 (García-Jerez et al. 2013), 用格林函数水平分量与垂 直分量的虚部的比值来模拟地脉动 $\mathrm{H} / \mathrm{V}$ 谱(SánchezSesma et al. 2011)。

随着面波理论的发展, 许多学者也通过理论面 波 $\mathrm{H} / \mathrm{V}$ 谱 (信号的水平分量与坚向分量之谱比) 来 模拟地脉动 $\mathrm{H} / \mathrm{V}$ 谱。但是计算理论面波谱比时需要 确定地脉动加载源或 $\mathrm{R} / \mathrm{L}$ 值 (地脉动水平分量的 Rayleigh 波与 Love 波的振幅比), 目前常用于确定地 脉动加载源或 $\mathrm{R} / \mathrm{L}$ 值的方法是通过地脉动台阵记录 提取面波频散曲线, 然后计算 $\mathrm{R} / \mathrm{L}$ 值。但如何基于 单点地脉动三分量记录获取 $\mathrm{R} / \mathrm{L}$ 值还需要进一步研 究。

\section{2. 理论方法}

在地表附近处，沿着地表传播的波被称为面波。 面波的种类很多, 其中最主要的是 Rayleigh 波和 Love 波。Rayleigh 波是英国物理学家 J. W. S. Rayleigh 在 1885 年首先通过理论推导出的, 后来也 在地震记录中得到证实, Rayleigh 波是由 $\mathrm{P}$ 波和 $\mathrm{SV}$ 波耦合形成的, 沿地表传播, 垂直于地面的平面内 作椭圆振动, 在地面处振幅最大, 随深度的增加,
波的振幅以指数形式衰减。Haskell(1953)对层状半 空间介质，在假设各层各向同性的基础上推导了层 状半空间中的 Rayleigh 波传播理论公式。Love 波是 英国物理学家 A. E. H. Love 在 1991 年首次提出的。 Love 波是 SH 波型的面波, 垂直于波的传播方向, 其振动方向于地面平行, Love 波形成的条件是浅层 $\mathrm{S}$ 波速度必须小于深层的, 即高速半空间上覆盖低 速水平地层。事实上, Haskell(1953)时就推导并给 出了层状半空间中的 Love 波传播理论公式和相应的 频散方程。这些理论更适于震源在远处时成层介质 由远场平面波体波（P-SV， SH）入射产生的 Rayleigh 面波和 Love 面波。由于地脉动问题的复杂 性, 某些情况下微震源可能处于成层介质的某一位 置。Harkrider(1964) 对此进行了分析。 Harkrider(1964)在 Haskell(1953)基础上发展并提出了 某一深度点源所引起的多阶面波的水平和垂直向功 率谱, 并提出了介质响应因子的概念。

本文使用 Harkrider(1964)基于面波在水平层状 介质传播理论提出的高阶面波的水平和垂直方向的 功率谱, 相关理论的具体推导过程如下。

第 $\mathrm{i}$ 个水平点源产生的第 $\mathrm{m}$ 阶 Rayleigh 波水平 和垂直方向的功率谱:

$$
\begin{aligned}
& P_{V R m i}^{H}(\omega) \\
& =\frac{1}{2} L_{H}^{2}(\omega) A_{R m}^{2}(\omega)\left(\frac{\dot{u}}{\dot{w}}\right)_{m}^{2}\left|H_{1}^{(2)}\left(k_{R m} r_{i}\right)\right|^{2} \\
& \exp \left(-2 h k_{R m} r_{i}\right) \\
& P_{H R m i}^{H}(\omega) \\
& =\frac{1}{2} L_{H}^{2}(\omega) A_{R m}^{2}(\omega)\left(\frac{\dot{u}}{\dot{w}}\right)_{m}^{4}\left|H_{0}^{(2)}\left(k_{R m} r_{i}\right)\right|^{2} \\
& \exp \left(-2 h k_{R m} r_{i}\right)
\end{aligned}
$$

式中，下标 $R$ 表示 Rayleigh 波， $A$ 为介质响应因子 (Harkrider，1964，1970)， $k$ 为波数, $h$ 为地层散射 阻尼系数, $r_{i}$ 为测点到点源的距离, $L_{H}$ 为测点到点 源的距离大于 Rayleigh 波波长或 Love 波波长时地表 处水平分量的随机分布, $\dot{u} / \dot{w}$ 为 Rayleigh 波在自由 表面处水平向与垂直向的速度比(Haskell, 1953), $H_{0}^{(2)}$ 和 $H_{1}^{(2)}$ 为第二类 Hankel 函数。第 $i$ 个水平点源产 生的第 $m$ 阶 Love 波水平方向的功率谱:

$$
\begin{aligned}
& P_{H L m i}^{H}(\omega) \\
& =\frac{1}{2} L_{H}^{2}(\omega) A_{L m}^{2}(\omega)\left|H_{0}^{(2)}\left(k_{L m} r_{i}\right)\right|^{2} \\
& \exp \left(-2 h k_{L m} r_{i}\right)
\end{aligned}
$$

式中, 下标 $L$ 分别表示 Love 波。第 $i$ 个垂直点源产 生的第 $m$ 阶 Rayleigh 波水平和垂直方向的功率谱: 


$$
\begin{aligned}
& P_{V R m i}^{V}(\omega) \\
& =L_{V}^{2}(\omega) A_{R m}^{2}(\omega)\left|H_{0}^{(2)}\left(k_{R m} r_{i}\right)\right|^{2} \\
& \exp \left(-2 h k_{R m} r_{i}\right) \\
& P_{H R m i}^{V}(\omega) \\
& =L_{V}^{2}(\omega) A_{R m}^{2}(\omega)\left(\frac{\dot{u}}{\dot{w}}\right)_{m}^{2}\left|H_{1}^{(2)}\left(k_{R m} r_{i}\right)\right|^{2} \\
& \exp \left(-2 h k_{R m} r_{i}\right)
\end{aligned}
$$

其中, $L_{V}$ 为测点到点源的距离大于 Rayleigh 波波长 时地表处垂直分量的随机分布。

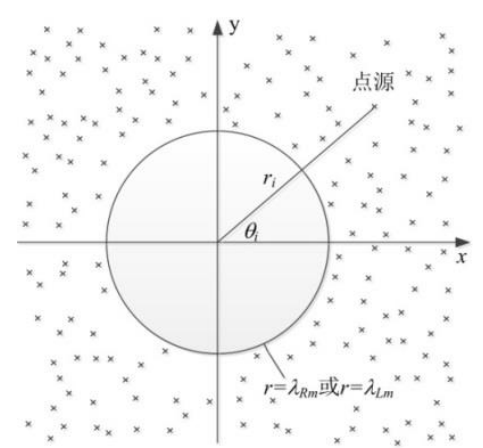

图 1 地脉动加载源模型图(Arai and Tokimatsu, 2000)

假定地脉动加载源是随机分布在地表的, 如图 1 所示。对于任意点源产生的面波与体波, 因为体 波的衰减比面波快, 所以在测点距点源距离大于一 倍面波波长时，可仅考虑面波的作用(Tamura, 1996)。 如图 1 中的圆点处所接收到的地脉动信号可以看作 半径为一倍面波波长圆以外的加载源所产生的面波 信号的集合。

Arai 和 Tokimatsu(2000)在假设所有点源的加载 阶段统计独立的基础上，将所有点源的各阶 Rayleigh 波和 Love 波合并, 可得到某观测点记录到 的所有激发点产生的面波在频率为 $\omega$ 时水平、垂直向 功率谱:

$$
\begin{aligned}
& P_{V R}^{V}(\omega) \\
& =\sum_{m=0}^{M} \int_{0}^{2 \pi} d \theta \int_{\lambda_{R m}}^{\infty} P_{V R m}^{V}(\omega) r_{i} d r_{i} \\
& P_{H R}^{V}(\omega) \\
& =\sum_{m=0}^{M} \int_{0}^{2 \pi} d \theta \int_{\lambda_{R m}}^{\infty} P_{H R m}^{V}(\omega) r_{i} d r_{i}
\end{aligned}
$$

$$
\begin{aligned}
& P_{H R}^{H}(\omega) \\
& =\sum_{m=0}^{M} \int_{0}^{2 \pi} d \theta \int_{\lambda_{R m}}^{\infty} P_{H R m}^{V}(\omega) r_{i} d r_{i} \\
& P_{V R}^{H}(\omega) \\
& =\sum_{m=0}^{M} \int_{0}^{2 \pi} d \theta \int_{\lambda_{R m}}^{\infty} P_{H R m}^{V}(\omega) r_{i} d r_{i} \\
& P_{H L}^{H}(\omega) \\
& =\sum_{m=0}^{M} \int_{0}^{2 \pi} d \theta \int_{\lambda_{L m}}^{\infty} P_{H L m}^{V}(\omega) r_{i} d r_{i}
\end{aligned}
$$

当激发点与接收点的距离 $r_{i}$ 大于一倍面波波长 时, Hankel 函数可表示为:

$$
\left|H_{n}^{(2)}(z)\right|^{2} \cong \frac{2}{\pi z}
$$

将其代入上述所有面波的水平、垂直向功率谱可得：

$$
\begin{gathered}
P_{V R}^{V}(\omega)=\kappa L_{V}^{2} \sum_{m=0}^{M}\left(\frac{A_{R m}}{k_{R m}}\right)^{2} \\
P_{H R}^{V}(\omega)=\kappa L_{V}^{2} \sum_{m=0}^{M}\left(\frac{A_{R m}}{k_{R m}}\right)^{2}\left(\frac{\dot{u}}{\dot{w}}\right)_{m}^{2} \\
P_{H R}^{H}(\omega)=\kappa \frac{L_{H}^{2}}{2} \sum_{m=0}^{M}\left(\frac{A_{R m}}{k_{R m}}\right)^{2}\left(\frac{\dot{u}}{\dot{w}}\right)_{m}^{4} \\
P_{V R}^{H}(\omega)=\kappa \frac{L_{H}^{2}}{2} \sum_{m=0}^{M}\left(\frac{A_{R m}}{k_{R m}}\right)^{2}\left(\frac{\dot{u}}{\dot{w}}\right)_{m}^{2} \\
P_{H L}^{H}(\omega)=\kappa \frac{L_{H}^{2}}{2} \sum_{m=0}^{M}\left(\frac{A_{L m}}{k_{L m}}\right)^{2}
\end{gathered}
$$

式中, $\kappa=(2 / h) e^{-4 \pi h}, \mathrm{~h}$ 表示土层的散射阻尼系 数。因此所有点源的各阶水平和垂直方向的功率谱 为:

$$
\begin{aligned}
& P_{V S}(\omega)=P_{V R}(\omega)=P_{V R}^{V}(\omega)+P_{V R}^{H}(\omega) \\
& =\kappa L_{V}^{2} \sum_{m=0}^{M}\left(\frac{A_{R m}}{k_{R m}}\right)^{2} \\
& +\kappa \frac{L_{H}^{2}}{2} \sum_{m=0}^{M}\left(\frac{A_{R m}}{k_{R m}}\right)^{2}\left(\frac{\dot{u}}{\dot{w}}\right)_{m}^{2}
\end{aligned}
$$




$$
\begin{gathered}
=\kappa L_{V}^{2} \sum_{m=0}^{M}\left(\frac{A_{R m}}{k_{R m}}\right)^{2}\left[1+\frac{\alpha^{2}}{2}\left(\frac{\dot{u}}{\dot{w}}\right)_{m}^{2}\right] \\
P_{H S}(\omega)=P_{H R}(\omega)+P_{H L}(\omega) \\
=P_{H R}^{V}(\omega)+P_{H R}^{H}(\omega)+P_{H L}^{H}(\omega) \\
=\kappa L_{V}^{2} \sum_{m=0}^{M}\left(\frac{A_{R m}}{k_{R m}}\right)^{2}\left(\frac{\dot{u}}{\dot{w}}\right)_{m}^{2} \\
+\kappa \frac{L_{H}^{2}}{2} \sum_{m=0}^{M}\left(\frac{A_{R m}}{k_{R m}}\right)^{2}\left(\frac{\dot{u}}{\dot{w}}\right)_{m}^{4} \\
+\kappa \frac{L_{H}^{2}}{2} \sum_{m=0}^{M}\left(\frac{A_{L m}}{k_{L m}}\right)^{2} \\
=\kappa L_{V}^{2} \sum_{m=0}^{M}\left\{\left(\frac{A_{R m}}{k_{R m}}\right)^{2}\left(\frac{\dot{u}}{\dot{w}}\right)_{m}^{2}[1\right. \\
\left.+\frac{\alpha^{2}}{2}\left(\frac{\dot{u}}{\dot{w}}\right)_{m}^{2}\right] \\
\left.+\frac{\alpha^{2}}{2}\left(\frac{A_{L m}}{k_{L m}}\right)^{2}\right\}
\end{gathered}
$$

式中, $\alpha$ 表示地脉动加载源, 其中 $\alpha=L_{H} / L_{V}$ 。则 Rayleigh 波 H/V 谱, Rayleigh 波和 Love 波振幅比 $\mathrm{R} / \mathrm{L}$ 以及面波 $\mathrm{H} / \mathrm{V}$ 谱为:

$$
\begin{aligned}
& (H / V)_{R}(\omega)=\sqrt{\frac{P_{H R}(\omega)}{P_{V R}(\omega)}} \\
& (R / L)(\omega)=\sqrt{\frac{P_{H R}(\omega)}{P_{H L}(\omega)}} \\
& (H / V)_{S}(\omega)=\sqrt{\frac{P_{H S}(\omega)}{P_{V S}(\omega)}} \\
& =\sqrt{\frac{P_{H R}(\omega)+P_{H L}(\omega)}{P_{V R}(\omega)}}
\end{aligned}
$$

\section{3. 玉溪两场地的地脉动 $\mathrm{H} / \mathrm{V}$ 谱模拟}

玉溪市城区位于该盆地西南部(如图 2 所示), 其 中场地 $\mathrm{A}$ 比场地 $\mathrm{B}$ 离城区更近。本文的研究场地是 $\mathrm{A}$ 和 $\mathrm{B}$ ，因为这两个场地既具有钻孔数据也有地脉 动数据。对场地 $\mathrm{A}$ 和 $\mathrm{B}$ 分别进行钻孔后使用 XG-I 悬挂式波速测井仪进行波速测试, 由钻孔测速得到 的浅层速度结构, 如图 3 所示。

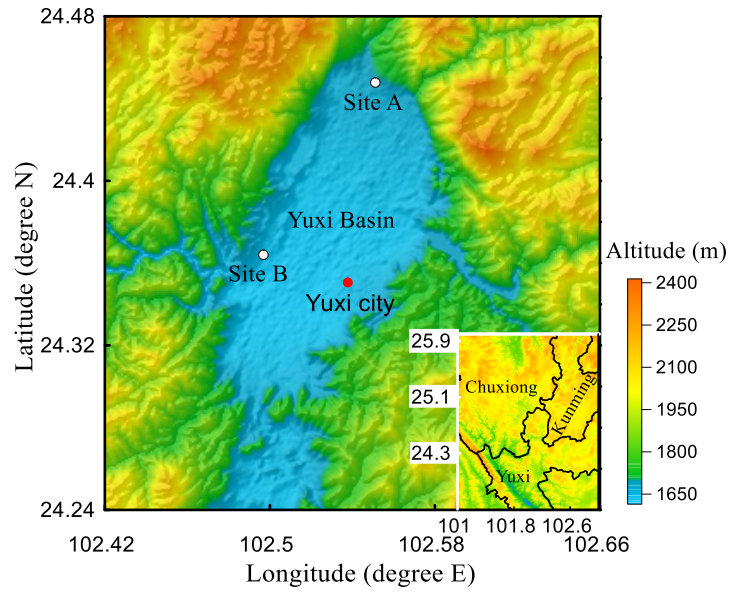

图 2 玉溪盆地的位置

（白色圆点代表场地位置, 红色圆点代表玉溪市城区.)
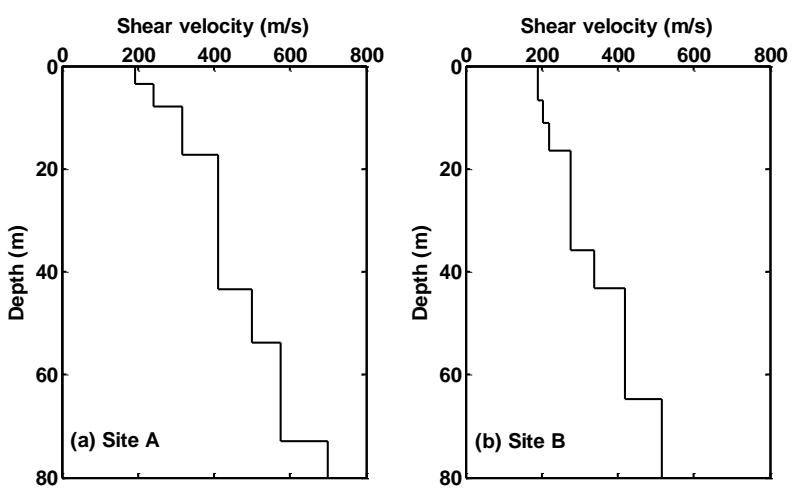

图 3 场地 $\mathrm{A}$ 和 $\mathrm{B}$ 钻孔的浅层剪切波速度剖面图

使用采样频率为 $8 \mathrm{~ms}$ 的三分量检波器, 对场地 $\mathrm{A}$ 和 $\mathrm{B}$ 分别进行地脉动观测。分别记录 3 段地脉动 信号, 每段时长约 $5 \mathrm{~min}$ 。在场地 $\mathrm{A}$ 和 $\mathrm{B}$ 的地脉动记 录中分别选取 10 段长度为 4096 个数据点的地脉动 记录, 用于计算地脉动 $H / V$ 谱 $(H / V)_{m}$ ：

$$
(H / V)_{m}(\omega)=\sqrt{\frac{P_{E W}(\omega)+P_{N S}(\omega)}{P_{U D}(\omega)}}
$$

式中, $P_{E W}$ 和 $P_{N S}$ 分别为东西和南北向傅里叶功率谱, $P_{U D}$ 为垂直向傅里叶功率谱。

利用面波理论模拟地脉动 $\mathrm{H} / \mathrm{V}$ 谱的前提是 $\alpha$ 值 已知。但基于地脉动加载源随机分布的假定得到面 波 $\mathrm{H} / \mathrm{V}$ 谱理论公式, 在实际场地测试中地脉动加载 源的 $\alpha$ 值无法测得。常规做法是先通过地脉动台站 数据利用空间自相关方法或频率-波数法确定 R/L 值, 通常认为或假定 $\mathrm{R} / \mathrm{L}$ 值与地脉动源的 $\alpha$ 值相当或相 等。从前人研究可知, 地脉动水平分量中 Love 波的 成分随场地条件和频率的不同有明显的波动, 且稳 定在 60\%-90\%之间(Köhler et al. 2007; Endrun 2011)。 
Arai 和 Tokimatsu (2000)发现不同场地条件和频率下 $\mathrm{R} / \mathrm{L}$ 值不同，但 $\mathrm{R} / \mathrm{L}$ 值稳定在 0.4-1.0 之间，在 0.1$5 \mathrm{~s}$ 周期内其平均值约为 0.7 。实际计算时，往往通过 水平方向上 Rayleigh 波和 Love 波振幅比来表示地脉 动的加载源。

当 $\mathrm{R} / \mathrm{L}(\alpha)$ 取不同的值时, 面波 $\mathrm{H} / \mathrm{V}$ 谱的曲 线形状大致相似, 但各曲线的峰值不同。例如, 当 $\alpha$ 值分别取 $0.4 、 0.7$ 和 1 时，面波 $\mathrm{H} / \mathrm{V}$ 谱的波峰频 率与地脉动 $\mathrm{H} / \mathrm{V}$ 谱的波峰频率相同, 但各面波 $\mathrm{H} / \mathrm{V}$ 谱曲线与实际地脉动观测的 $\mathrm{H} / \mathrm{V}$ 谱曲线的振幅是不 相同的。因此，面波 $\mathrm{H} / \mathrm{V}$ 谱的波峰频率与地脉动加 载源的 $\alpha$ 值无关, 而面波 $\mathrm{H} / \mathrm{V}$ 谱的振幅与地脉动加 载源的 $\alpha$ 值有关。地脉动的 $\mathrm{H} / \mathrm{V}$ 谱曲线反演速度结 构的最佳频率范围, 位于地脉动的 $\mathrm{H} / \mathrm{V}$ 谱曲线的峰 值和谷值频率之间。

本文定义在共振频率 $\omega_{0}$ 处, 使面波 $\mathrm{H} / \mathrm{V}$ 谱与地 脉动 $\mathrm{H} / \mathrm{V}$ 谱的振幅偏差在 0.2 以内的 $\mathrm{R} / \mathrm{L}$ 值为最优 $R / L$ 值。当 $R / L$ 值取最优值时, 面波 $H / V$ 谱模拟地 脉动 $\mathrm{H} / \mathrm{V}$ 谱的效果较好(如图 4 所示), 此时, 场地 $\mathrm{A}$ 的 $\alpha$ 值为 0.75 , 场地 $\mathrm{B}$ 的 $\alpha$ 值为 0.65 。本文将量 场地的 $\mathrm{R} / \mathrm{L}(\alpha)$ 最优值视为真实值。

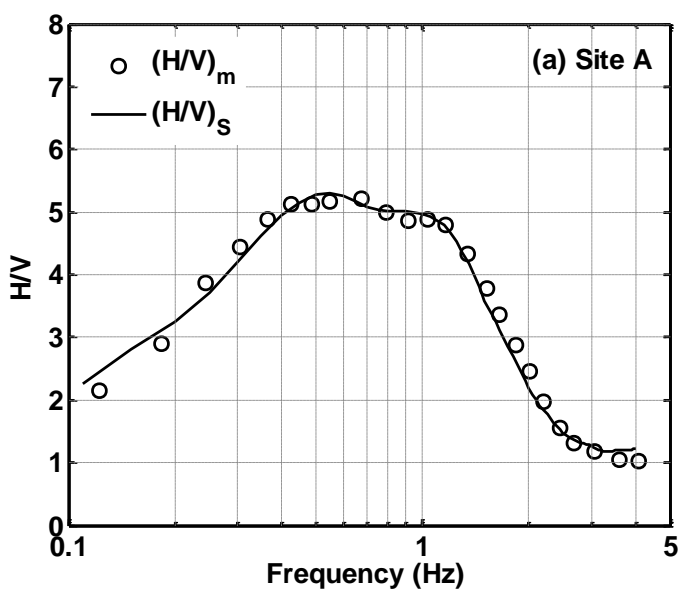

(A)

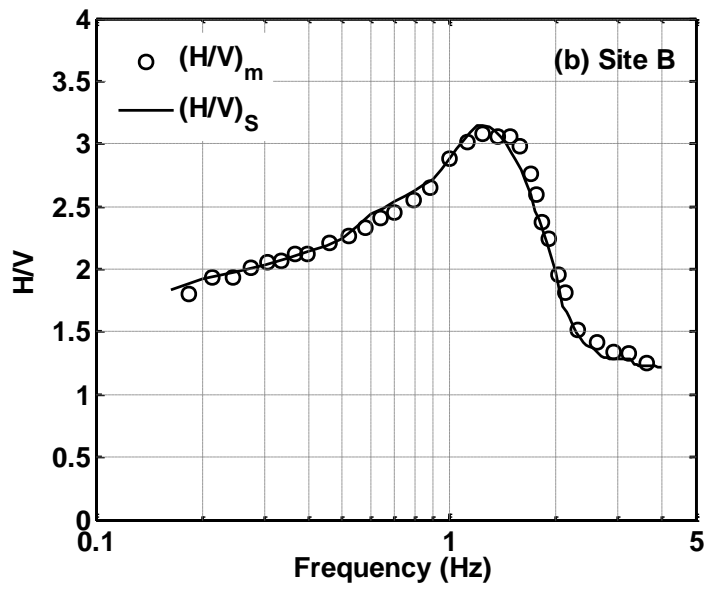

(B)

图 4 场地 $\mathrm{A}$ 和 $\mathrm{B}$ 的地脉动 $\mathrm{H} / \mathrm{V}$ 谱及理论面波 $\mathrm{H} / \mathrm{V}$ 谱
图 4 给出了场地 $\mathrm{A}$ 和 $\mathrm{B}$ 的地脉动 $\mathrm{H} / \mathrm{V}$ 谱及理论 面波 $\mathrm{H} / \mathrm{V}$ 谱曲线。从图 4 中可看出, 场地 $\mathrm{A}$ 和 $\mathrm{B}$ 的 基阶共振频率分别为 $0.55 \mathrm{~Hz}$ 和 $1.5 \mathrm{~Hz}$, 即共振周期 (卓越周期/自振周期) 为 $1.82 \mathrm{~s}$ 和 $0.67 \mathrm{~s}$ 。因此, 本 文在分析场地 $\mathrm{A}$ 和 $\mathrm{B}$ 的地脉动数据时选用的频率范 围是 $0.1-5 \mathrm{~Hz}$ 之间是合理的。由于场地 $\mathrm{A}$ 的基岩埋 深(402m) 比场地 B 的基岩埋深(189m)大, 所以场地 $\mathrm{A}$ 的基阶共振频率比场地 $\mathrm{B}$ 的基阶共振频率小。这 些场地的卓越周期参数与场地覆盖层厚度和等效剪 切波速所计算的结果是基本一致的。

\section{4. 结论与讨论}

我们使用理论面波 $\mathrm{H} / \mathrm{V}$ 谱模拟了地脉动 $\mathrm{H} / \mathrm{V}$ 谱 (Zhang, Chen, et al., 2018)。模拟了玉溪盆地 $\mathrm{A}$ 和 $\mathrm{B}$ 两个场地的地脉动 $\mathrm{H} / \mathrm{V}$ 谱曲线。虽然面波 $\mathrm{H} / \mathrm{V}$ 谱曲 线的幅值与 $\alpha$ 值, 即地脉动加载源的水平与垂直分 量的比值, 有一定关系, 但面波 $\mathrm{H} / \mathrm{V}$ 谱曲线的峰值 频率与 $\alpha$ 值无关。本文给出了玉溪两场地的 $\alpha$ 值。 此外, 面波 $\mathrm{H} / \mathrm{V}$ 谱曲线在峰值频率处对 $\mathrm{R} / \mathrm{L}$ 值最为 敏感。为减小利用地脉动的 $\mathrm{H} / \mathrm{V}$ 谱曲线反演地下速 度结构的误差, 在地脉动 $\mathrm{H} / \mathrm{V}$ 谱的反演中不应考虑 在近峰值频率处的面波 $\mathrm{H} / \mathrm{V}$ 谱曲线, 因为使用面波 $\mathrm{H} / \mathrm{V}$ 谱曲线模拟地脉动的 $\mathrm{H} / \mathrm{V}$ 谱曲线, 在峰值频率 处误差较大。

本文讨论了 $\mathrm{R} / \mathrm{L}$ 取不同值时，理论面波 $\mathrm{H} / \mathrm{V}$ 谱 与地脉动 $\mathrm{H} / \mathrm{V}$ 谱的拟合程度, 并对面波 $\mathrm{H} / \mathrm{V}$ 谱的特 性进行讨论。并且, 地脉动的 $\mathrm{H} / \mathrm{V}$ 谱曲线反演速度 结构的最佳频率范围位于地脉动的 $\mathrm{H} / \mathrm{V}$ 谱曲线的峰 值和谷值频率之间。

\section{致谢}

感谢国家重点研发计划（2019YFC1509403, 2017YFC1500205）、国家自然科学基金（51678537, 51978633,51278470 ) 和研究所基金（DQJB19B06） 的共同资助。感谢匿名专家提出的有益于提升研究 的审稿意见。

\section{参考文献}

[1] 袁一凡,田启文, 工程地震学 $[\mathrm{M}]$, 北京: 地震出版 社, 2012 .

[2] 陶夏新, 师黎静, 董连成, 2002. 中日地脉动台 阵联合观测 $[J]$. 世界地震工程, (2): pp24-31.

[3] 陶夏新,刘曾武,郭明珠,等,2001. 工程场地条件评 定中的地脉动研究 [J]. 地震工程与工程振动, (4).pp18-23 
[4] 郭迅,黄玉龙,袁一凡,2000. 香港场地类别划分的初 步研究[J].世界地震工程,(1).pp51-60.

[5] 杨学林,吴世明,周亚刚,1997. 利用短周期地脉动推 断深层地基 $\mathrm{S}$ 波速度 $[\mathrm{J}]$.振动工程学报,(2).pp5359.

[6] 王振东,1986. 微动的空间自相关法及其实用技术 [J].物探与化探, (2).pp123.

[7] 郭明珠,谢礼立,凌贤长,2004. 弹性介质面波地脉动 单点谱比法研究 [J]. 岩土工程学报,26(4):pp450453.

[8] 师黎静, 陶夏新,2007.地脉动方法最新研究进展 [J]. 地震工程与工程振动,27(6),pp30-37.

[9] 董连成,陶夏新,师黎静,李广影,2008. 利用地脉动 台阵记录反演场地浅层 Vs 结构 [J]. 岩土力学, 29(2): pp553-556.

[10] 师黎静, 苏茜, 刘宇实等,2016. 厦门本岛近地表三 维速度结构建模研究 [J]. 振动与冲击,35(16): pp43-48.

[11] Zhang Z, Chen XL, Gao MT, et al, 2018. Simulation of the microtremor $\mathrm{H} / \mathrm{V}$ spectrum based on the theory of surface wave propagation in a layered half-space[J]. Acta Geophysica, (66): pp121-130.

[12] Picozzi M, Parolai S, Richwalski SM, 2005. Joint inversion of $\mathrm{H} / \mathrm{V}$ ratios and dispersion curves from seismic noise: Estimating the S-wave velocity of bedrock[J]. Geophysical Research Letters 32(11):pp339-357.

[13] Bonnefoy-Claudet S, Cornou C, Bard PY, Cotton F, Moczo P, Kristek J, Fa“h D, 2006. H/V ratio: a tool for site effects evaluation. Results from 1-D noise simulations. Geophys J Int 167(2):pp827837.

[14] Carcione JM, Picotti S, Francese R, Giorgi M, Pettenati F, 2017. Effect of soil and bedrock anelasticity on the S-wave amplification function[J]. Geophys J Int 208:pp424-431.

[15] Fäh D, Kind F, Giardini D, 2003. Inversion of local s-wave velocity structures from average $\mathrm{H} / \mathrm{V}$ ratios, and their use for the estimation of site-effects[J]. Journal of Seismology 7(4):pp449-467.

[16] Yamanaka H, Takemura M, Ishida H, Niwa M., 1994. Characteristics of long-period microtremors and their applicability in exploration of deep sedimentary layers[J]. Bull Seismol Soc Am 84 (6):pp1831-1841.

[17] García-Jerez A, Luzón F, Sánchez-Sesma FJ, et al, 2013. Diffuse elastic wavefield within a simple crustal model[J]. Journal of Geophysical Researchsolid Earth 118(10):pp5577-5595.

[18] Sánchez-Sesma F J, Rodríguez M, IturraránViveros U, et al, 2011. A theory for microtremor $\mathrm{H} / \mathrm{V}$ spectral ratio: Application for a layered medium[J]. Geophys J Int, 186(1): pp221-225.

[19] Haskell N A, 1953. The dispersion of surface waves on multilayered media[J]. Bull Seismol Soc Am, 43(1): pp17-34.

[20] Harkrider D G, 1964. Surface waves in multilayered elastic media. 1. Rayleigh and Love waves from buried sources in a multilayered elastic half-space[J]. Bull Seismol Soc Am, 54(2): pp627679.

[21] Harkrider D G, 1970. Surface waves in multilayered elastic media. Part II. Higher mode spectra and spectral ratios from point sources in plane layered Earth models[J]. Bull Seismol Soc Am, 60(6): pp1937-1987.

[22] Tamura S, 1996. Comparison of body and Rayleigh wave displacements generated by a vertical point force on a layered elastic medium. In the 11th World conference on earthquake engineering, Acapulco, Mexico, 23-28 June 1996

[23] Arai H, Tokimatsu K, 2000. Effects of Rayleigh and Love waves on microtremor $\mathrm{H} / \mathrm{V}$ spectra. In: the 12th world conference on earthquake engineering, Auckland, New Zealand, 30 January4February 2000.

[24] Köhler A, Ohrnberger M, Scherbaum F, et al, 2007. Assessing the reliability of the modified threecomponent spatial autocorrelation technique[J]. Geophysical Journal International 168(2):pp779796.

[25] Endrun B, 2011. Love wave contribution to the ambient vibration $\mathrm{H} / \mathrm{V}$ amplitude peak observed with array measurements[J]. Journal of Seismology 15(3):pp443-472. 